\title{
D'une préservation volontaire de l'environnement par les entreprises à une préservation ordonnée ?
}

\author{
Gurvan Branellec \\ Docteur en droit \\ Professeur associé Brest Business School \\ Responsable du Programme Bachelor in International Management \\ Chercheur associé UMR AMURE
}

\begin{abstract}
Résumé :
Le Secrétaire général de l'ONU avait souligné lors du Sommet de Johannesburg que, face aux lenteurs des actions gouvernementales, les problèmes environnementaux et sociaux ne peuvent être résolus qu'en mobilisant les entreprises privées.

La question se pose donc de savoir si la préservation de l'environnement peut être laissée aux entreprises qui deviendraient volontairement des acteurs responsables de leur environnement (Blin-Franchomme et Desbarats, 2009, p. 19) ou si les pouvoirs publics doivent intervenir en la matière?

Pour répondre à cette question, l'étude mobilisera à la fois le cadre juridique mais également la littérature qu'elle soit juridique ou en sciences de gestion. La démonstration sera faite que nous assistons à un double mouvement en la matière puisque nous évoluons d'une prise en compte volontaire mais désordonnée par les entreprises de l'environnement (1) à une prise en compte ordonnée par le droit (2). La conclusion proposera toutefois de dépasser cette opposition traditionnellement décrite dans la littérature portant sur la responsabilité sociétale des entreprises entre un droit dur qui émane des Etats et des démarches volontaires qui proviennent des entreprises. Il sera plutôt fait le constat d'un système juridique dans lequel la frontière entre ces différentes normes s'atténue au profit d'un continuum de normes plus ou moins contraignantes qui présentent l'avantage de permettre plus de souplesse et d'efficacité.
\end{abstract}

Mots clés :

Entreprise, responsabilité sociétale des entreprises, environnement, droit souple.

\begin{abstract}
During the Johannesburg Summit, the Secretary General of the United Nations insisted on the need to mobilize private firms in response to the slowness of government actions, if one wanted to solve social and environmental problems.

The question therefore arises of whether environmental preservation can be left to businesses which would voluntarily become responsible actors of their environment (Blin-Franchomme et Desbarats, 2009, p. 19) or whether public authorities should get involved.

In order to answer this question, the present study draws both on the legal framework and the literature in law and management sciences. It demonstrates that a shift can be observed from a voluntary but disorganized taking into account of the environment by businesses (1) to an organized taking into account by the law (2). The conclusion suggests that the traditional opposition described in the corporate social responsibility literature between hard law
\end{abstract}


originating from the States, and the conscious efforts originating from businesses, should be overcome. A legal system appears within which the frontier between these two norms becomes thinner in favor of a continuum of more or less binding norms which have the advantage of being more flexible and efficient.

\section{Key words}

Business, Corporate social responsibility, environment, soft law.

L'entreprise a une responsabilité sociale ou/et sociétale. Elle doit répondre aux intérêts de la collectivité et intégrer mais surtout respecter un certain nombre de valeurs dont la durabilité du développement (on peut également citer l'éthique des affaires ou la citoyenneté).

Le développement durable, défini par le rapport Brundtland comme «le développement qui répond aux besoins du présent sans compromettre la capacité des générations futures à répondre aux leurs $»^{1}$, fait désormais figure de boussole pour la croissance économique. L'entreprise est évidemment un acteur essentiel pour atteindre une croissance économique durable. La citoyenneté des entreprises s'est ainsi muée en responsabilité sociétale des entreprises (Chauveau et Rosé, 2003, 252), celles-ci devant s'efforcer de concilier progrès économique, social et respect de l'environnement. Cette reconnaissance par les entreprises d'une responsabilité à l'égard de leur environnement se développe surtout du fait des pressions de la société civile (Desbarats, 2008, $\mathrm{n}^{\circ} 32$ ) et se caractérise essentiellement par le caractère volontaire des démarches. Le Secrétaire général de l'ONU a d'ailleurs souligné lors du Sommet de Johannesburg que, face aux lenteurs des actions gouvernementales, «c'est seulement en mobilisant le secteur privé que nous ferons des progrès significatifs. Le sommet de Johannesburg est une occasion historique pour fédérer les règles des entreprises et du développement durable. Les problèmes environnementaux et sociaux ne peuvent être résolus qu'en mobilisant les entreprises privées ».

Le droit français contient des dispositions règlementant les activités économiques, principalement industrielles, et visant à limiter leur impact sur l'environnement (installations classées, gestion des déchets, régimes d'autorisations administratives...). Ces dispositions ne s'appliquent toutefois pas à toutes les entreprises et constituent davantage des contraintes administratives qu'une véritable règlementation juridique globale des entreprises visant à une préservation de l'environnement. Face à l'absence d'une règlementation complète et structurée les entreprises se sont emparées de la question environnementale et se présentent de plus en plus comme un acteur responsable qui protège l'environnement.

Les entreprises mettent ainsi en place des chartes environnementales, codes de conduite, labels, systèmes de certification environnementale, accords environnementaux... qui sont autant de «signes de qualités privés » (Blin-Franchomme, 2009, $\mathrm{n}^{\circ}$ 40) qui se développent en dehors de la contrainte de la loi. Ces actions peuvent avoir des motivations diverses: potentialité des éco-activités, nécessité de préserver durablement l'activité de l'entreprise, prévention des risques juridiques ou économiques, vision utilitariste de cette préservation afin d'en faire un outil de communication... Les domaines concernés par ces engagements ainsi que les valeurs consacrées, sont d'une grande diversité. Leur forme ainsi que les formules employées le sont tout autant. Elles peuvent aller de formules floues qui comportent des engagements imprécis jusqu'à des adjonctions aux règlements internes des entreprises

\footnotetext{
${ }^{1}$ Rapport déposé le 27 avril 1987 par la Commission mondiale sur l'environnement et le développement (CMED) intitulé «Notre avenir à tous».
} 
(Pereira, 2009, p. 30) ou des engagements pris par des entreprises ou branches sectorielles. Un risque existe, que l'engagement de l'entreprise en matière de préservation de l'environnement se fasse a minima voire même qu'il soit factice.

Ces initiatives privées produisent des conséquences juridiques puisque la jurisprudence peut sanctionner la déloyauté d'une démarche ou le non-respect d'un engagement. Ainsi, l'Etat ne se désintéresse pas de ces questions. Son intervention dans les relations entre les entreprises et l'environnement ne se cantonne toutefois pas à une mise en jeu possible de la responsabilité des entreprises devant les tribunaux. Un processus de normalisation et de régulation juridique est aujourd'hui en marche même s'il n'existe pas pour le moment de cadre juridique achevé en la matière.

La question se pose donc de savoir si la préservation de l'environnement peut être laissée aux entreprises qui deviendraient volontairement des acteurs responsables de leur environnement (Blin-Franchomme et Desbarats, 2009, p. 19) ou si les pouvoirs publics doivent intervenir en la matière?

Pour répondre à cette question, l'étude mobilisera à la fois le cadre juridique mais également la littérature qu'elle soit juridique ou en sciences de gestion. La démonstration sera faite que nous assistons à un double mouvement en la matière puisque nous évoluons d'une prise en compte volontaire mais désordonnée par les entreprises de l'environnement (1) à une prise en compte ordonnée par le droit (2). La conclusion proposera toutefois de dépasser cette opposition traditionnellement décrite dans la littérature portant sur la responsabilité sociétale des entreprises entre un droit dur qui émane des Etats et des démarches volontaires qui proviennent des entreprises. Il sera plutôt fait le constat d'un système juridique dans lequel la frontière entre ces différentes normes s'atténue au profit d'un continuum de normes plus ou moins contraignantes qui présentent l'avantage de permettre plus de souplesse et d'efficacité.

\section{D'une préservation désordonnée par les entreprises de l'environnement}

La prise en compte par les entreprises des enjeux environnementaux est liée à des motivations très diverses (1.1) et elle se traduit de différentes manières (1.2).

\section{1) Une préservation en réponse à des motivations diverses}

L'idée que l'entreprise a une responsabilité à l'égard de son environnement est assez ancienne (mécénat, politique sociale de grandes entreprises) mais elle se développe aujourd'hui à cause des pressions sociales, environnementales et économiques (Desbarats, 2008, $\mathrm{n}^{\circ}$ 32). En effet, le comportement d'une entreprise n'est pas uniquement susceptible d'affecter les actionnaires ou dirigeants, il peut également concerner d'autres parties prenantes internes (salariés) ou externes (consommateurs, créanciers, clients, citoyens...).

L'idée de responsabilité sociale va émerger au début du XIXe siècle aux Etats-Unis lors de l'apparition de grandes entreprises à actionnariat dispersé. Ces entreprises vont donc connaître une dissociation entre l'exercice du pouvoir et la détention du capital, l'entreprise va donc devenir une institution à part entière qui interagit avec son environnement (Acquier et Aggeri, 2008, p. 133 et 134). Le concept de responsabilité sociale de l'entreprise (RSE) a alors été développé afin de faire entrer les parties prenantes dans la gouvernance d'entreprise grâce aux valeurs sociales, environnementales et éthiques.

L'application pratique de la RSE dans le monde des entreprises va être plus tardive puisque c'est en 1984 que des multinationales américaines vont adopter des codes de conduite et vont imposer à leurs sous-traitants le respect de normes minimales s'agissant des conditions de 
traitement de leurs salariés (Robin-Olivier, Beauchard et de La Garanderie, 2011, p. 395).

Cette démarche est de prime abord une démarche éthique. Les entreprises vont ainsi reconnaître qu'au-delà de ce que la loi impose il existe des freins à leur activité (Trébulle, 2002, p.2). L'entreprise prend donc pleinement conscience qu'elle est un acteur incontournable de notre écosystème car elle est la cellule économique essentielle. Elle accepte alors de dépasser des normes et éventuellement de sacrifier une partie de leurs avantages ou bénéfices au profit d'un intérêt supérieur: sa contribution au développement durable. L'entreprise devient citoyenne grâce à cette démarche éthique. L'entreprise devenant à cet égard un acteur politique dans ses relations avec son environnement (Cazal, 2008, p.13). Cette démarche éthique n'est pas toutefois totalement ou toujours désintéressée tant il est vraisemblable que «l'éthique paye » (Trébulle, 2002, p. 4). En effet, cette démarche peut parfois être initiée pour éviter des mises en jeu de leur responsabilité et réduire le risque lié aux impacts négatifs que les activités de l'entreprise peuvent avoir sur l'environnement et le risque de réparation qui en résulte. Elle peut également être développée afin que l'entreprise ne laisse pas le champ libre à des concurrents plus en avance sur ces questions. Elle peut être le fruit d'une stratégie marketing visant à améliorer leur image de marque. Les consommateurs amènent aussi les entreprises à adopter des démarches de prise en compte de leur responsabilité environnementale en intégrant des considérations environnementales dans leurs comportements de consommation. Les entreprises vont alors réaliser un arbitrage entre le coût et la satisfaction de ces demandes d'une plus grande responsabilité environnementale (Guillon, 2006, p.43).

Il peut enfin s'agir d'une démarche intéressée des entreprises qui sont incitées à prendre en compte leur responsabilité sociétale sous la pression des investisseurs ou des prêteurs qui sont de plus en plus attentifs à la performance extra-financière des entreprises.

S'agissant des prêteurs, les établissements de crédit intègrent de plus en plus fréquemment les paramètres environnementaux dans les critères d'évaluation de leurs clients (Trébulle, 2003, p.5). Ceux-ci ont en effet compris que l'environnement était un facteur de risques qui ne peut être minimisé que lorsque l'entreprise a pleinement mesuré les impacts avérés ou potentiels de son activité sur l'environnement et qu'elle engage des actions pour réduire ou maîtriser ces impacts.

En ce qui concerne les investisseurs, l'engagement sociétal des entreprises constitue de plus en plus à l'heure actuelle un critère d'investissement. Les fonds ISR en sont un bon exemple. L'investissement socialement responsable est un investissement individuel ou collectif effectué selon des critères sociaux, environnementaux ou éthiques. Ces fonds sont issus de la pratique. Le premier ISR de l'économie moderne vient des Etats-Unis et remonte au 10 août 1971. À cette date, deux pasteurs de l'Église méthodiste, Luther Tyson et Jack Corbett, lancent le "Pax World Fund" pour investir dans les entreprises non liées à l'armement ${ }^{2}$. Ils font leur apparition en France en 1983 lorsque le Comité catholique contre la faim et pour le développement lance avec le Crédit Coopératif, le premier fonds commun de placement de partage Faim et développement. L'investissement dans un fonds ISR bénéficie à présent du soutien des Nations Unies qui ont consacré cet investissement en l'élevant à l'échelle mondiale (Malecki, 2009). Cet investissement s'est considérablement développé au point qu'en 2010, selon Novethic (centre de recherche sur la Responsabilité Sociale et Environnementale des entreprises et l'Investissement Socialement Responsable créé par la Caisse des dépôts), les encours ISR dans les fonds ouverts distribués en France s'élèvent à près de 65 milliards d'Euros (avec une hausse annuelle de l'ordre de 35\%). Au-delà du formidable succès de ces initiatives privées, se pose un problème de lisibilité et de contrôle de ces fonds dits «verts». En 2001, les acteurs de l'ISR (investisseurs institutionnels,

\footnotetext{
${ }^{2}$ http://www.paxworld.com/about-pax-world/history
} 
gestionnaires d'actifs, agences de notations extra-financières, courtiers...) ont commencé à se pencher sur ces questions au sein du Forum pour l'Investissement Responsable. Paris Europlace a de son côté publié en mai 2008 onze recommandations pour favoriser l'investissement socialement responsable. Dans sa première proposition, il en appelle à une intervention du législateur qu'il estime nécessaire. Il s'agirait « de demander aux investisseurs institutionnels d'élaborer un reporting annuel public sur la manière dont ils ont pris en compte les critères ESG dans leurs décisions de placement $»^{3}$.

Ces différents éléments attestent donc qu'une démarche de responsabilité volontaire peut être bénéfique en matière d'image mais également en termes financiers. Cette démarche volontaire peut prendre des formes diverses.

\section{2) Une préservation de l'environnement prenant des formes diverses}

Cette prise en compte par les entreprises de leur responsabilité à l'égard de l'environnement se caractérise surtout par des démarches volontaires. Cette intégration volontaire par les entreprises des préoccupations sociales et écologiques à leur activité commerciale peut être considérée comme étant la marque d'une «société contractuelle » (Robin-Olivier, Beauchard et de La Garanderie, 2011, p. 395), une approche civique du rapport de l'entreprise au droit. On vient toutefois de voir que cette entreprise «moralisée » reste toutefois arrimée à une approche individualiste et économiquement intéressée (Cazal, 2008, p.21) de ses rapports avec son environnement.

Les entreprises se dotent ainsi de chartes environnementales, codes de conduite, codes de déontologie... Il s'agit de « signes de qualités privés » (Blin-Franchomme, 2009, n 40) qui se développent en dehors de la contrainte de la loi. Les règles issues de la responsabilité sociale des entreprises reposent sur le double postulat du volontariat pour ce qui est de l'adhésion, et de l'absence de contrainte pour ce qui est de la mise en œuvre (Trébulle, 2002, p. 10).

Ces initiatives privées se rencontrent dans des domaines variés : accords environnementaux (éco-labels, réduction des emballages...), engagements sociaux (développement durable, commerce équitable...), règles de conduite financière ou éthique (rémunération des dirigeants, non-discrimination, amélioration des conditions de travail des salariés)... Les domaines concernés, ainsi que les valeurs consacrées, sont d'une grande diversité. La forme de ces engagements et les formules employées le sont tout autant. Ces démarches volontaires peuvent aller de formules floues qui comportent des engagements imprécis jusqu'à des adjonctions aux règlements internes des entreprises (Pereira, 2009, p. 30) ou des engagements pris par des entreprises ou branches sectorielles qui vont produire des conséquences juridiques.

Ces chartes et codes de conduite permettent aux entreprises de formaliser et de communiquer sur une démarche responsable à l'égard de leur environnement. Leur multiplication (Desbarats, 2003, $\mathrm{n}^{\circ} 112$ ) conduit à s'interroger sur leur portée juridique.

La plupart des chartes et codes éthiques sont des actes unilatéraux ; ils n'ont donc pas de valeur contractuelle même s'ils peuvent matérialiser un engagement unilatéral (BlinFranchomme, 2013, n78) de l'entreprise qui pourra être invoqué contre elle (l'action pourra ainsi être intentée sur le fondement de la responsabilité civile délictuelle (Deumier, 2009, 122)). Par ailleurs, comme le constate Madame Blin-Franchomme «ces engagements de bonne pratique ne visent en réalité qu'assez rarement à aller au-delà des exigences légales » (Blin-Franchomme, 2009, $\mathrm{n}^{\circ}$ 40). Une étude réalisée par l'OCDE (Organisation de coopération et de développement économique) en 2000 sur 246 codes de conduite montre

\footnotetext{
${ }^{3}$ http://www.agefi.fr/etude/rapport_isr_europlace.pdf
} 
ainsi que ces codes contiennent souvent des engagements formulés dans des termes généraux ou imprécis (OCDE, 2000). Les entreprises se sont effectivement empressées de communiquer sur le fait qu'elles prenaient en compte leur responsabilité sociale. Cette communication leur permettant principalement « de moderniser et de dynamiser l'image de l'entreprise, d'accroître sa notoriété et sa légitimité »(Garric, Léglise et Point, 2006, p.13). Pourtant ces entreprises ne sont pas toutes aussi environnementalement responsables qu'elles veulent bien le dire. On parle ainsi de green washing ou d'écoblanchiment. L'organisation CorpWatch définit l'écoblanchiment comme « un comportement d'entreprise nocif du point de vue social ou environnemental qui tente de préserver et d'étendre son marché en se présentant comme un ami de l'environnement et un leader dans le combat pour éradiquer la pauvreté » (Roglev, Beckhard et Becker, 2012, n67). La Communication de la Commission européenne au Parlement européen, au Conseil, au Comité économique et social européen et au Comité des régions intitulée "Responsabilité sociale des entreprises: une nouvelle stratégie de l'Union européenne pour la période 2011-2014» (Comm. UE, n681 final, 25 oct. 2011) parle d'ailleurs de comportement «irresponsable» de certaines entreprises qui exagèrent leurs mérites dans le domaine environnemental ou social. Ainsi, l'entreprise Renault dans une publicité pour son modèle de véhicule électrique Zoé diffusé sur son site Internet présentait celui-ci comme écologique. Le Jury de Déontologie Publicitaire ${ }^{4}$ a considéré que «l'allégation selon laquelle un véhicule électrique serait «écologique » ou « écolo » est de nature à induire en erreur le public quant aux propriétés du produit vendu » (JDP, Renaut Zoé, 263/13).

La diffusion d'information portant sur la prise en compte de l'environnement par les entreprises pose ainsi le problème de la comparabilité et de la fiabilité de celles-ci même si des initiatives ont été menées en ce sens (Trébulle, 2002, p. 8) et notamment les lignes directrices du Global Reporting Initiative (qui restent toutefois une démarche volontaire).

Il ne faudrait pas pour autant nier toute efficacité aux démarches volontaires initiées par les entreprises. Un bon exemple peut être pris avec les recommandations adoptées par l'Autorité de régulation professionnelle de la publicité (ARPP). Afin de lutter contre l'écoblanchiment, cette autorité a publié les recommandations «arguments écologiques » et «développement durable ». Les normes contenues dans ces recommandations ont pour objectif d'empêcher l'utilisation abusive de l'argument écologique et la promotion de comportements contraires aux principes de développement durable. Les professionnels de la publicité se sont engagés le 3 décembre 2007 à systématiquement consulter l'ARPP avant la diffusion d'une campagne incluant des arguments écologiques (Hanse et Grelier-Lenain, 2008, p.4). L'ARPP amène les publicitaires ainsi que les annonceurs à modifier des publicités qui ne respectent pas ces recommandations. Le Conseil de l'éthique publicitaire considère d'ailleurs que ce système d'autorégulation par le biais de ces règles déontologiques adoptées par les professionnels de la publicité « étaient anticipatrices et uniques en Europe. Allant au-delà des obligations légales, elles contribuent, sans nul doute, aux bons résultats obtenus à ce jour en conciliant le principe fondamental de liberté d'expression et l'impératif de responsabilité sociale. L'application de ces règles déontologiques, qui relèvent d'une démarche de responsabilité, doit permettre d'éviter les dérives » (Hanse et Grelier-Lenain, 2008, p.8).

Les entreprises cherchent donc à donner une visibilité à leur démarche. Pour ce faire, elles font régulièrement appel à la certification d'organismes tiers qui attestent du respect par l'entreprise de normes privées. Les normes privées sont très prisées par les entreprises. Elles

\footnotetext{
${ }^{4}$ Le Jury de Déontologie Publicitaire est une instance indépendante rattachée à l'Autorité de régulation professionnelle de la publicité (ARPP) qui a pour mission de statuer sur les plaintes du public à l'encontre de publicités diffusées. Elle est composée de 9 membres impartiaux nommés par le Conseil d'administration de l'ARPP.
} 
peuvent s'appliquer aussi bien à des activités techniques qu'au système de management ou à la responsabilité sociale de l'entreprise. Au niveau national, le guide français de référence SD 21000 ( $c f$. le site AFNOR) permet de structurer l'entreprise autour des préoccupations sociétales. Au niveau international, l'Organisation Internationale de Normalisation (OIN) a déjà créé des référentiels en matière sociale ou environnementale comme la famille ISO 14000. Par exemple la formulation «consommation réduite d'énergie » est précisément définie dans la norme ISO 14021 (article 7.9.1 de la norme ISO 14021 relative aux autodéclarations environnementales 1999). Cet organisme a également élaboré la méta-norme ISO 26000 sur la responsabilité sociétale. Ce référentiel d'engagement (Cadet, 2010) formalise des lignes directrices, d'application facultative, qui définissent la responsabilité sociale de toute organisation.

Ce caractère volontaire de la prise en compte par les entreprises de leur responsabilité environnementale ne signifie pas que l'Etat se désintéresse de cette question. L'analyse du droit - spécialement français - montre que la responsabilité sociétale et environnementale s'exerce aujourd'hui dans un cadre juridique dont l'élaboration n'est vraisemblablement pas achevée (Desbarats, 2008, $\mathrm{n}^{\circ} 32$ ).

\section{A une préservation de l'environnement ordonnée aux entreprises par le droit}

Les entreprises ne sont plus les seules à se préoccuper de leur impact sur l'environnement. Les pouvoirs publics se saisissent également de ces questions que ce soit pour encadrer les démarches volontaires des entreprises (2.1) ou pour leur prescrire de nouveaux comportements ou mettre à leur charge de nouvelles obligations (2.2).

\subsection{Une intervention du droit pour encadrer les démarches volontaires}

L'intérêt des pouvoirs publics pour les questions de responsabilité environnementale des entreprises peut s'expliquer par plusieurs raisons. Il y a d'abord le fait que les valeurs mises en avant par les entreprises dans le cadre de leurs démarches renvoient à des considérations d'intérêt général ; les pouvoirs publics ne pouvaient alors continuer à s'en désintéresser. Les engagements affichés par les entreprises peuvent également favoriser des abus; ils ne pouvaient donc échapper à la sphère juridique. Ensuite, car leur multiplication fait craindre que ces chartes et codes, développés dans le cadre de ces démarches, ne soient que des artéfacts publicitaires, «que le quantitatif ou le marketing prime sur la qualité » (Delga, 2005, $\mathrm{n}^{\circ}$ 84). Il y a enfin le fait que la prévention par les entreprises du risque environnemental et sa gestion par une intégration en amont ne sont pas toujours efficaces. L'Etat doit donc intervenir en aval pour sanctionner ces atteintes et obliger les entreprises à réparer leurs atteintes à l'environnement.

L'interventionnisme du législateur ou du juge dans les questions de RSE est ainsi nécessaire ne serait-ce que pour sanctionner des entreprises qui annoncent un projet social ou environnemental fictif et portent ainsi atteinte à l'image d'entreprises qui mettent en place des démarches sincères. Ces différentes raisons ont donc poussé les pouvoirs publics, législateur ou juge, à «attirer dans le giron du droit certains comportements éthiques » (Desbarats, 2008, $\mathrm{n}^{\circ} 32$ ).

L'interventionnisme du législateur ou du juge dans les questions de RSE prend plusieurs formes qui vont d'une simple incitation à l'encadrement des démarches volontaires des entreprises.

Les pouvoirs publics peuvent avoir un rôle d'incitateur en poussant les entreprises à agir en menaçant d'utiliser l'outil législatif (l'OCDE a souligné la nécessité de «menaces crédibles 
d'une règlementation contraignante $»^{5}$; un bon exemple peut être trouvé dans un autre domaine : il s'agit de la rémunération des dirigeants de sociétés cotées ${ }^{6}$ ). Ces incitations peuvent également être économiques, ainsi des dispositifs favorisant la prise en compte du développement durable et de la protection de l'environnement ont été progressivement intégrés dans le droit des marchés publics français à la faveur de la directive européenne 2004/18/CE du 31 mars 2004. Le Code des marchés publics a autorisé en 2004 les considérations environnementales comme critères d'attribution, à condition qu'elles soient en rapport avec l'objet du marché. Cette démarche a été étendue en 2006 puisque le Code des marchés publics impose la prise en compte du développement durable dès la définition des besoins (art. 5) et prévoit que les spécifications techniques de l'appel d'offres puissent inclure des caractéristiques environnementales (art. 6). Les clauses sociales et environnementales peuvent également influer sur les conditions d'exécution (art. 14) ainsi que les performances en matière de protection de l'environnement qui font partie des critères d'attribution des marchés (art. 53). Enfin, le décret $n^{\circ} 2011-1000$ du 25 août 2011 introduit la possibilité de conclure des contrats globaux de performance, dans lesquels le titulaire s'engage notamment en matière d'efficacité énergétique ou d'incidence écologique.

Les pouvoirs publics peuvent également avoir un rôle de partenaire dans le cadre d'une «corégulation » que ce soit par le biais de l'ouverture d'une réflexion ou d'une concertation (signature de conventions avec des branches sectorielles dans le cadre du Grenelle de l'environnement).

Ils peuvent encore surveiller voire donner force obligatoire aux engagements des entreprises. Ainsi, les Principes pour l'investissement responsable (PRI), initiés par le Programme des Nations Unies pour l'environnement et auxquels les établissements financiers adhérent librement, sont à l'origine des démarches volontaires. Pourtant, une fois intégrés par l'entreprise, notamment dans un cadre contractuel, les engagements pris deviendront obligatoires et leur non-respect pourra être sanctionné par les tribunaux (Trébulle, 2007, p. 12). Il en sera notamment ainsi lorsque l'entreprise aura signifié à ses cocontractants l'importance de ses engagements environnementaux ou éthiques, ceux-ci intègreront donc le champ contractuel et tout manquement sera assimilable à une inexécution contractuelle qui permettra d'engager la responsabilité contractuelle de l'entreprise (Ferrari, 2012, $\mathrm{n}^{\circ} 76$ ). Un juge pourra même faire produire des effets à un accord conclu entre des parties alors même que les parties avaient annoncé leur intention de ne pas rendre cet accord obligatoire (Oppetit, 1979, $\mathrm{n}^{\circ} 13$ ). Une démarche volontaire de l'entreprise ne signifie pas que celle-ci est facultative (Trébulle, 2002, p. 6). Certains auteurs considèrent même qu'une démarche RSE d'une entreprise permettrait de transformer une obligation naturelle en une obligation civile. Dans le cadre d'une obligation naturelle la personne n'est pas juridiquement liée, elle s'engage pour des raisons morales. Si l'obligation devient civile elle peut faire l'objet d'une exécution forcée. Une entreprise qui s'engage à adopter un comportement écologiquement

\footnotetext{
${ }^{5}$ OCDE, « Les approches volontaires dans les politiques de l'environnement : efficacité et combinaison avec d'autres instruments d'intervention, Paris, 2003, $161 \mathrm{p}$.

${ }^{6}$ Le gouvernement a ainsi plusieurs fois menacé d'intervenir, notamment sur la question des éléments de rémunération exceptionnel tels que les actions gratuites, les stocks-options ou les bonus, par le biais de l'outil législatif. Les stocks-options qui auraient dû être versés aux dirigeants de la Société Générale et à ceux de GDFSuez ayant relancé la polémique. Plusieurs pistes sont envisagées : taxation plus lourde des stock-options, interdiction des stock-options pendant une certaine durée, lier la distribution de bonus aux risques pris et aux résultats induits par ces risques ainsi qu'à un horizon de temps correspondant aux risques pris... Le gouvernement a d'ailleurs utilisé l'outil contraignant en adoptant le décret n 2009-348 du 30 mars 2009 qui s'applique aux entreprises ayant été aidées par l'Etat (six grandes banques françaises et deux groupes automobiles). Celui-ci permet d'encadrer les rémunérations variables qui devront être établies à partir de critères de performance précis et limités dans le temps. Le décret précise également qu'en cas d'indemnité de départ « celle-ci est fixée à un montant inférieur à deux années de rémunération ».
} 
responsable au-delà des seules exigences règlementaires, à condition que cet engagement soit suffisamment précis, pourrait s'exposer à une mise en jeu de sa responsabilité si elle ne les respecte pas. Ces engagements précis peuvent concerner la gouvernance mais aussi l'action de l'entreprise en matière sociale et environnementale. Une entreprise qui ne serait pas responsable juridiquement d'une pollution pourrait ainsi s'engager à assurer la dépollution d'un site sans qu'elle ne puisse revenir sur ses engagements y compris si le coût de pollution s'avère finalement trop élevé et risque d'obérer ses capacités de croissance (Poracchia et Martin, 2012, p. 475).

Il en résulte que des engagements volontaires pris par une entreprise peuvent se traduire en obligations sanctionnées juridiquement.

Sur le plan des pratiques commerciales, la loi n ${ }^{\circ} 2008-3$ du 3 janvier $2008^{7}$, complétée par la loi $\mathrm{n}^{\circ}$ 2008-776 du 4 août 2008, permet de sanctionner la déloyauté d'une démarche affichant une préoccupation environnementale qui pourrait être qualifiée de pratique commerciale trompeuse $^{8}$. Le juge avait, de toute façon, déjà la possibilité de sanctionner ces comportements sur le fondement de l'action en concurrence déloyale.

Les acteurs civils ne s'arrêtent plus aux portes de l'entreprise mais poussent celle-ci à agir voire contrôlent les actions de celle-ci (Blin-Franchomme, 2010, $\mathrm{n}^{\circ}{ }^{52}$ ). Les associations agréées de protection de l'environnement ont ainsi la possibilité d'agir en cas d'infraction aux dispositions législatives relatives à la protection de la nature et de l'environnement. Cette possibilité d'action a été étendue par l'article 229 de la loi du 12 juillet 2010 ( $\left.\mathrm{n}^{\circ} 2010-788\right)$ portant engagement national pour l'environnement (dite loi Grenelle 2) qui leur permet également de poursuivre des allégations environnementales infondées lorsque « les pratiques commerciales et les publicités trompeuses ou de nature à induire en erreur quand ces pratiques et publicités comportent des indications environnementales ».

Les pouvoirs publics peuvent enfin mettre à la charge des entreprises de nouvelles obligations ou contraintes afin que celles-ci développent des comportements responsables à l'égard de l'environnement.

\subsection{Une intervention du droit pour créer de nouvelles obligations à la charge des entreprises}

Il vient d'être démontré que la RSE se caractérisait principalement par le caractère volontaire des démarches initiées par les entreprises. Ces démarches ont souvent eu un effet de préparation puisqu'elles ont facilité une prise de conscience qui a débouché sur des interventions du législateur (Trébulle, 2002, p. 9).

Ainsi la loi $\mathrm{n}^{\mathrm{0}} 2001-420$ du 15 mai 2001 relative aux nouvelles régulations économiques (loi NRE) est une consécration légale des démarches volontaires de diffusion d'informations initiées par les entreprises. Le législateur a toutefois renforcé, encadré et étendu ces démarches volontaires. La loi NRE a d'abord imposé une transparence environnementale aux

\footnotetext{
${ }^{7}$ Cette loi, dite « loi Chatel » a transposé la directive du Parlement européen et du Conseil $\mathrm{n}^{\mathrm{o}}$ 2005/29 du 11 mai 2005 relative aux pratiques commerciales déloyales des entreprises.

${ }^{8}$ La loi LME du 4 août 2008 énonce ainsi, dans un nouvel article L. 121-1-1 du Code de la consommation, que constitue des présomptions de pratiques commerciales trompeuses, le fait "pour un professionnel, de se prétendre signataire d'un code de conduite alors qu'il ne l'est pas; d'afficher un certificat, un label de qualité ou un équivalent sans avoir obtenu l'autorisation nécessaire; d'affirmer qu'un code de conduite a reçu l'approbation d'un organisme public ou privé alors que ce n'est pas le cas (...)».
} 
entreprises cotées sur un marché réglementé en leur enjoignant de faire figurer dans un rapport des informations environnementales et sociales. La loi Grenelle 2 et le décret $\mathrm{n}^{\circ} 2012$ 557 du 24 avril 2012 relatif aux obligations de transparence des entreprises en matière sociale et environnementale ont ensuite étendu cette obligation aux SA et SCA qui dépassent des seuils fixés à 100 millions d'euros pour le total du bilan, 100 millions d'euros pour le montant net du chiffre d'affaires et à 500 pour le nombre moyen de salariés permanents employés au cours de l'exercice (le seuil relatif à l'exercice est cumulatif avec celui du chiffre d'affaire ou celui du total bilan). Le projet de loi visait à l'origine les entreprises de plus de 250 salariés au chiffre d'affaires de plus de 50 millions d'euros ou au total de bilan de plus de 43 millions d'euros, ce qui était assez logique car il s'agit du seuil utilisé par l'Union européenne pour distinguer les PME des plus grandes entreprises. Le lobbying actif du MEDEF (Mouvement des Entreprises de France) et de l'AFEP (Association française des Entreprises privées), deux organisations patronales, a permis de rehausser ce seuil (Cuzacq, 2013, p. 15). La mise en œuvre de ce dispositif est différenciée et échelonnée (François, 2012, p. 607) : les sociétés non cotées dont le total du bilan ou le montant net du chiffre d'affaires dépasse 1 milliard d'euros et dont le nombre moyen de salariés permanents employés au cours de l'exercice est supérieur à 5000 sont tenue d'établir ce rapport pour les exercices ouverts après le 31 décembre 2011. Les sociétés non cotées dont le total du bilan ou le montant net du chiffre d'affaires dépasse 400 millions d'euros et dont le nombre moyen de salariés permanents employés au cours de l'exercice est supérieur à 2000 l'établiront pour les exercices ouverts après le 31 décembre 2012. Quant aux sociétés non cotées dont le total du bilan ou le montant net du chiffre d'affaires dépasse 100 millions d'euros et dont le nombre moyen de salariés permanents employés au cours de l'exercice est supérieur à 500, elles n'auront l'obligation d'établir le rapport extra-financier que pour les exercices ouverts après le 31 décembre 2013. Il s'agit ici de l'application d'un principe « rendre compte pour mieux prendre en compte » (Blin-Franchomme, 2008, $\mathrm{n}^{\circ}$ 32). Le rapport de gestion doit exposer les actions menées et les orientations prises par la société afin qu'elle remplisse ses engagements sociétaux en faveur du développement durable. Les entreprises doivent donner de l'information sur des thèmes dont la liste est prévue par le décret du 24 avril 2012. Une première liste concerne toutes les sociétés assujetties à l'obligation de reporting et une seconde liste uniquement les sociétés dont les titres sont admis aux négociations sur un marché règlementé. Une société peut tout à fait ne pas renseigner certains thèmes à condition dans ce cas de se justifier. Il s'agit d'une transposition du principe anglo-saxon comply or explain. Ce dispositif a pour effet de permettre aux parties prenantes de prendre connaissance des informations sociales et environnementales qui concernent l'activité des sociétés. Elle doit permettre également de comparer de manière objective les performances extra-financières des entreprises. S'agissant des informations environnementales, le rapport doit préciser «l'organisation de la société pour prendre en compte les questions environnementales » ainsi que les moyens consacrés à la prévention des risques environnementaux et des pollutions (article R. 225-105-1 I $2^{\circ}$ a). Le rapport devra également indiquer les mesures de prévention, de réduction et de réparation des rejets dans l'air, l'eau et le sol affectant gravement l'environnement ainsi que la prise en compte des nuisances sonores et toute autre forme de pollution spécifique à son activité. Le rapport devra également détailler la consommation d'eau, de matières premières et celle d'énergie et les mesures prises pour améliorer l'efficacité dans leur utilisation ainsi que le recours aux énergies renouvelables et l'utilisation des sols. Le rapport devra également faire mention des rejets de gaz à effet de serre ainsi que des mesures prises pour préserver ou développer la biodiversité. Ces mesures peuvent paraître prescriptives, elles ne le sont pas. Elles constituent toutefois une avancée car elles contraignent les entreprises à communiquer sur leur prise en compte ou non prise en compte de ces questions.

Cette loi reste toutefois une lex imperfecta (Cuzacq, 2013, p. 15) car les parties prenantes 
peuvent tout à fait ne pas intégrer ces informations dans leurs arbitrages. Par ailleurs, les informations peuvent n'être que lacunaires car les entreprises peuvent très bien ne pas renseigner certains thèmes, elles ont également la possibilité de définir elles-mêmes le contenu des indicateurs.

Malgré ce constat la loi Grenelle 2 augmente la contrainte qui pèse sur les entreprises car la délivrance de ces informations extra-financières est obligatoire (ou tout du moins la justification de la non-délivrance de ces informations dans le cadre du explain). Les tiers peuvent d'ailleurs tout à fait consulter le rapport de gestion. Pour garantir la sincérité des informations communiquées la loi Grenelle 2 met en place un audit des informations sociales et environnementales puisqu'elle prévoit que ces informations feront l'objet «d'une vérification par un organisme tiers indépendant » (article L. 225-102-1 alinéa 7 du Code de commerce). L'auditeur extra-financier est désigné par les organismes accrédités par le Comité français d'accréditation. Ce dispositif d'audit est toutefois perfectible. D'une part, car il ne prévoit pas de sanction spécifique dans l'hypothèse d'une absence de nomination de l'auditeur par le dirigeant. Ensuite, la loi n'a pas prévu que les tiers aient le droit de consulter ce rapport de l'auditeur extra-financier ce qui constitue une atteinte non-négligeable au principe de transparence en matière sociétale. Enfin, l'auditeur ne va vérifier qu'une partie de l'information extra-financière qui sera délivrée par l'entreprise. En effet, les différents supports de communication internes ou externes dans lesquels peuvent figurer des informations environnementales ou sociales (par exemple un rapport RSE ou développement durable) ne sont pas inclues dans son périmètre de contrôle.

Les pouvoirs publics renforcent également la responsabilité des entreprises à l'égard des dommages qu'elles peuvent occasionner à l'environnement. La Charte de l'environnement du 24 juin 2004, à qui le Parlement a conféré une valeur constitutionnelle par la loi constitutionnelle du 28 février 2005, prévoyait déjà le principe du «pollueur-payeur » qui n'a pas qu'un principe réparateur mais doit permettre également une prévention des dommages environnementaux et vise aussi à internaliser les coûts de la pollution au sein des entreprises. La loi Grenelle 2 est venue renforcer le principe du «pollueur-payeur » en prévoyant que les entreprises pourront se voir imposer des mesures à la fois préventives et curatives en cas d'atteintes graves causées par leurs activités à l'environnement. Les juges reconnaissent d'ailleurs, depuis l'arrêt Erika (Cass. Crim. 25 sept. 2012, n¹0-82.938) le «préjudice environnemental » qui permet de réparer le dommage à l'environnement lui-même ou plus précisément aux «biens environnementaux» (Blin-Franchomme, 2013, $\mathrm{n}^{\circ} 78$ ) indépendamment du dommage subi par des personnes physiques ou morales (Martin, 1995, p. 115). Ce préjudice environnemental recouvre de façon classique les atteintes à l'environnement lui-même (atteinte à un intérêt collectif) mais pourrait également recouvrir les atteintes que la dégradation environnementale induit en matière de services écologiques rendus par la nature aux hommes (services culturels, d'ordre récréatifs, esthétiques, ou économiques tels que l'eau douce, la bioénergie, la pollinisation...). Cette dernière approche dite «écosystémique » n'est pour le moment pas retenue par les tribunaux. Il est pourtant tout à fait possible que ce type de préjudice soit admis dans le futur. Les entreprises ont d'ores et déjà tout intérêt à intégrer cette approche systémique puisqu'elles peuvent être dépendantes de ces services environnementaux dans le cadre de son activité, elles auront donc tout intérêt à limiter leurs impacts sur l'environnement et à veiller à sa protection voire à sa restauration.

Cette loi prend donc en compte les initiatives privées issues des entreprises. Elle va plus loin puisque la loi Grenelle 2 a créé une responsabilité des sociétés mères à l'égard de leurs filiales en liquidation judiciaire (repris à l'article L. 512-17 du Code de l'environnement). Cette disposition fait suite à l'impunité dont avait pu bénéficier le Groupe Metaleurop suite à l'importante pollution du site de sa filiale Metaleurop Nord qui avait été mise en liquidation judiciaire ce qui avait contraint la collectivité à prendre en charge la dépollution du site. $\mathrm{Ce}$ 
texte met ainsi à la charge des sociétés mères tout ou partie du financement des mesures de remise en état du ou des sites en fin d'activité, à l'initiative du liquidateur, du ministère public ou du représentant de l'Etat, lorsque «l'existence d'une faute caractérisée commise par la société mère qui a contribué à une insuffisance d'actif de la filiale» est établie. Pour permettre aux sociétés mères de pouvoir éviter cette situation, la loi Grenelle 2 a institué un mécanisme prophylactique leur permettant la prise en charge volontaire des obligations de prévention des dommages causés à l'environnement.

\section{Conclusion}

En définitive, il apparaît que la prise en compte de l'environnement n'est plus en marge du droit mais bien directement visée par celui-ci.

Ce droit se caractérise par le caractère important des démarches volontaires. Cette approche «micro-juridique» de la règlementation (Salin et Laine, 2003, $\mathrm{n}^{\circ}$ 45-46) est d'ailleurs privilégiée par 1'OCDE et l'Union européenne (Maljean-Dubois, 2008, p. 37) car elle laisse une marge de manœuvre importante aux entreprises sur les modalités de prise en compte de leur environnement. Ce droit est un droit souple au sens de la définition qu'en donne le Conseil d'Etat ${ }^{9}$ en ce qu'il modifie ou oriente les comportements sans créer par lui-même de droits ou d'obligations comme le droit traditionnel (Constitution, lois, décrets...) mais en présentant un certain degré de formalisation et de structuration. Il présente l'avantage «d'enrichir la palette des pouvoirs publics ${ }^{10}$ qui n'avaient auparavant le choix qu'entre un droit dur et contraignant et le non-droit. Ce droit d'une plus grande flexibilité normative a des frontières plus poreuses et ouvertes sur les phénomènes sociaux et les pratiques des acteurs économiques. En effet, ce droit qui propose des modèles d'action est « largement codéterminé par le juge et ses destinataires » (Thibierge, 2003, p. 599 et s.).

Pourtant ce droit souple, et notamment les démarches volontaires des entreprises en matière de préservation de l'environnement, doit être parfois repris par les pouvoirs publics. En effet, l'usage des instruments de droit souple par les entreprises exige un contrôle accru des juridictions (Renaud, 2013, 1601) car il fait courir un risque de privatisation de la règle de droit ou d'instrumentalisation de celle-ci ou il risque de favoriser des discours auto-légitimant (Thibierge, 2003, p. 599 et s.).

L'objectif est ainsi de développer en la matière une «échelle de normativité graduée ». Ainsi, pour les risques d'atteintes les plus graves à l'environnement il est nécessaire de combiner les démarches volontaires et leur incitation par les pouvoirs publics avec des procédés contraignants. La norme juridique n'est donc plus seulement un ordre de contrainte mais devient également » un modèle pour agir et une référence pour juger de l'action » (Martin, 2008, p. 317).

Il nous faut donc constater le caractère complexe de la relation entre les entreprises et l'environnement du fait de l'enchevêtrement entre des démarches volontaires issues du bas (des entreprises) et des interventions venues du sommet (l'Etat). Il est possible de parler à cet égard de «boucle double » de la RSE (Robin-Ollivier, Beauchard et de La Garanderie, 2011, p. 395): bottom-up pour les engagements volontaires des entreprises et top down pour l'action des pouvoirs publics. Le croisement de ces deux démarches permet une hybridation des cultures juridiques et un enrichissement mutuel. Il est dès lors possible de développer l'idée d'un droit formant un système, composé d'éléments réunis par un réseau de relations plus ou moins complexes (Delmas-Marty, 2006, p. 26). Ce système présente les

\footnotetext{
${ }^{9}$ Conseil d'Etat, Etude annuelle 2013 « Droit souple », Documentation Française.

${ }^{10}$ Ibid.
} 
caractéristiques des systèmes juridiques (Gassin, 1980, p. 353) en ce qu'il est ouvert (il entretient des relations étroites avec des intervenants économiques et politiques) et imparfait en ce qu'il contient des normes contradictoires. Cette branche du droit tend vers une hybridation s'agissant de ses sources car le droit n'est ni construit à partir du haut, ni élaboré de façon spontanée par le bas, mais est plutôt construit par touches horizontales successives de normes de valeurs différentes, de provenances diverses, ayant un champ d'application dissemblable.

Acquier, A. et Aggeri, F. 2008. Une généalogie de la pensée managériale sur la RSE, Revue française de gestion, $\mathrm{n}^{\circ} 180$, p. 131-157.

Beauchard, R., de La Garanderie, D. et Robin-Olivier S. 2011. La responsabilité sociale de l'entreprise (RSE), Revue de droit du travail, p. 395.

Blin-Franchomme, M.-P. 2013. Code de conduite : Attention à l'écart de conduite !, Revue Lamy droit des affaires, $\mathrm{n}^{\circ} 78$.

Blin-Franchomme, M.-P. et Desbarats, I. 2009. L'entreprise bioacteur, Bulletin du droit de l'environnement industriel, p. 19.

Blin-Franchomme, M.-P. 2008. Entreprise et responsabilité : aperçu de quelques avancées récentes du développement durable dans la vie des affaires, Revue Lamy droit des affaires, $\mathrm{n}^{\circ}$ 32.

Blin-Franchomme, M.-P. 2009. De quelques éléments de régulation des démarches volontaires en matière de RSE... », Revue Lamy droit des affaires, $\mathrm{n}^{\circ} 40$.

Blin-Franchomme, M.-P. 2010. L'entreprise et ses parties prenantes externes : l'aiguillon des associations de protection de l'environnement, Revue Lamy droit des affaires, $\mathrm{n}^{\circ} 52$.

Blin-Franchomme, M.-P. 2013. Le préjudice environnemental dans tous ses états, Revue Lamy droit des affaires, $\mathrm{n}^{\circ} 78$.

Borgetto, M. 2006. L'économie sociale et solidaire : quelle place pour le droit ? , in Les dynamiques de l'économie sociale et solidaire, sous la direction de Jean-Noël Chopart, Guy Neyret et Daniel Rault 2006, La Découverte.

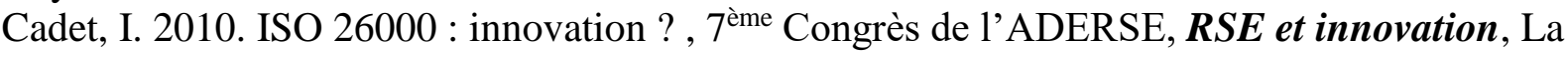
Rochelle, 24-26 mars 2010.

Cazal, D, 2008. Parties prenantes et RSE : des enjeux sociopolitiques au-delà des contrats, Revue de l'organisation responsable, vol. 3, p. 12 à 23.

Chauveau, A. et Rosé, J-J. 2003. L'entreprise responsable, Editions d'Organisation.

Cuzacq, N. 2013. Les nouveautés issues du décret $n^{\circ} 2012-557$ du 24 avril 2012 relatif aux obligations de transparence des entreprises en matière sociale et environnementale, Revue de droit du travail, p. 5 et s.

Delmas-Marty, M. 2006. Le pluralisme ordonné, Les forces imaginantes du droit (III), Seuil 2006, p. 26.

Desbarats, I. 2008. Regard sur un instrument majeur de la gouvernance d'entreprise : quid de la nature juridique des codes éthiques ?, Revue Lamy droit des affaires, $\mathrm{n}^{\circ} 32,2008$.

Delga, J. 2005. Propos iconoclastes sur l'éthique d'entreprise: Une analyse juridique de l'éthique d'entreprise au regard de la mondialisation, Revue Lamy droit des affaires, $\mathrm{n}^{\circ} 84$.

Deumier, P. 2009. La réception du droit souple. In Le droit souple, Dalloz.

Ferrari, J. 2012. La société mère peut-elle voir sa responsabilité engagée dans le cadre de la RSE ?, Revue Lamy droit des affaires, $\mathrm{n}^{\circ} 76$.

François, B. 2012. Reporting RSE : commentaire du décret $n^{\circ} 2012-557$ du 24 avril 2012, Revue des sociétés, p. 607.

Garric, N., Léglise, I. et Point, S. 2006. Le rapport RSE, outil de légitimation ? Le cas 
TOTAL à la lecture de l'analyse de discours, Revue de l'organisation responsable, ${ }^{\circ} 2, \mathrm{p} .5$ 19.

Gassin, R. 1980. Système et droit, Revue de la recherche juridique, $\mathrm{n}^{\circ} 11, \mathrm{p} .353$ et $\mathrm{s}$.

Guillon, B. 2006. La responsabilisation environnementale et la profession pétrolière française : une démarche progressive mariant la valorisation des savoirs-faire et le lobbying, Revue de l'organisation responsable, vol. 1, p. 38 à 47.

Guyon, Y. et Coquereau, G. 1984. Les réformes apportées... par la loi du $1^{\mathrm{er}}$ mars $1984, \boldsymbol{J C P}$ éd. E, I, 1984, nº 13426.

Hanse, F. et Grelier-Lenain, C. 2008. Les règles déontologiques du développement durable, Gazette du Palais, n $^{\circ} 341$, p. 3 et s.

Malecki, C. 2009. L'investissement socialement responsable: quelques problématiques actuelles », Revue Lamy droit des affaires, $\mathrm{n}^{\circ} 40$.

Maljean-Dubois, S. 2008. Quel droit pour l'environnement, Hachette.

Martin, G.-J. 1995. Réflexions sur la définition du dommage à l'environnement : le dommage écologique pur, in Droit et environnement, PUAM.

Martin, G.-J. 2009. Conclusion générale, in Les approches volontaires et le droit de l'environnement, N. Hervé-Fournereau (dir.), PUR.

OCDE, Groupe de travail du Comité des échanges : Les codes de conduite des entreprises Etude approfondie de leur contenu : TD/TC/WP (99)56/FINAL du 13 juin 2000.

Oppetit, B. 1979. L'engagement d'honneur, Dalloz, 1979, chronique 106.

Pereira, B. 2009. Chartes et codes de conduite : le paradoxe éthique, Revue Management et avenir, $\mathrm{n}^{\circ} 21$, p. 30 .

Poracchia, D.et Martin, D. 2012. Regard sur l'intérêt social, Revue des sociétés, p. 475.

Renaud, A. 2013. Le Conseil d'Etat célèbre le droit souple, Semaine Sociale Lamy, 1601.

Roglev, L., Beckhard, J.et Becker, V. 2012. Responsabilité sociale des entreprises : une compliance à l'européenne? Revue Lamy droit des affaires $n^{\circ} 67$.

Salin, P. et Laine, M. 2003. Le mythe de la transparence imposée, JCP E , 6-13 novembre $\mathbf{n}^{\circ}$ 45-46.

Thieberge, C. 2003. Le droit souple : Réflexion sur les textures du droit, Revue Trimestrielle de Droit Civil, 2003, p. 599.

Trébulle, F.-G. 2003. (mis à jour en 2012). Responsabilité sociale des entreprises, Répertoire sociétés Dalloz.

Trébulle, F.-G. 2004. La comptabilisation de l'environnement, Droit des sociétés, p. 9.

Trébulle, F.-G. 2007. Entreprise et développement durable, JCL Environnement, p. 12. 